07

\title{
Моделирование детектора видимого и ближнего инфракрасного электромагнитного излучения на искусственном алмазе
}

\author{
(C) B.A. Кукушкин \\ Федеральный исследовательский центр Институт прикладной фризики РАН, \\ 603950 Нижний Новгород, Россия \\ Нижегородский государственный университет им. Н.И. Лобачевского, \\ 603950 Нижний Новгород, Россия \\ e-mail: vakuk@appl.sci-nnov.ru
}

(Поступило в Редакцию 9 мая 2018 г. В окончательной редакции 17 июля 2018 г.)

Разработан метод расчета вольт-амперной характеристики детектора электромагнитного излучения видимого и ближнего инфракрасного диапазона длин волн на искусственном алмазе при вертикальной геометрии протекания через него электрического тока. Метод применим в случае, когда длина свободного пробега носителей заряда в алмазе меньше масштаба изменения их концентрации. На основе этого метода определены зависимость тока от напряжения, а также распределения концентрации носителей заряда и электростатического потенциала для конкретного варианта такого фотодетектора, основанного на поликристаллической алмазной пленке, состоящей из монокристаллов нанометровых размеров, допированных бором.

DOI: 10.21883/JTF.2019.02.47080.181-18

\section{Введение}

Алмаз, вследствие своей высокой химической и радиационной стойкости, является перспективной основой фотодетекторов для атомной промышленности и космических исследований. Вследствие большой ширины запрещенной зоны энергий $(5.5 \mathrm{eV})$ он обычно используется для создания фотодетекторов ультрафиолетового и мягкого рентгеновского диапазона длин волн [1-3]. Фотодетекторы важного для телекоммуникации ближнего инфракрасного диапазона на алмазе могут быть основаны на изменении его сопротивления при облучении благодаря генерации дополнительных носителей заряда за счет переходов примесная зона-зона [4] или нагрева [5]. Однако вследствие трудности контролирования и воспроизведения параметров примесных зон и инерционности тепловых эффектов такие устройства не получили большого распространения.

В то же время ближний инфракрасный фотодетектор на алмазе может быть основан на другом эффекте, хорошо известном в оптоэлектронике [6]. Он заключается в рождении носителей заряда (конкретно дырок) не в самом алмазе, а в прилежащем к нему слое металла, служащем одновременно и одним из контактов с внешней цепью. Вследствие тонкости этого слоя (типичная толщина порядка $10 \mathrm{~nm}$ ) рожденные в нем детектируемым излучением дырки успевают до рекомбинации с электронами диффундировать в алмаз. Там они ускоряются электрическим полем прилежащей к контакту области пространственного заряда и создают таким образом фототок. Очевидно, что наименьшая энергия фотонов, которые можно зарегистрировать таким способом, должна быть порядка разницы энергии Ферми в металле и верхнего края валентной зоны алмаза на его границе с металлом. Как известно [7], для контакта ал- маза с металлами эта разница может отвечать видимому и ближнему инфракрасному диапазону длин волн.

Основанный на этом эффекте ближний инфракрасный фотодетектор на монокристаллическом алмазе, осажденном из газовой фазы (CVD) на монокристаллическую алмазную подложку, был реализован авторами [8]. Однако интересным представляется использование для этой цели также и поликристаллического алмаза, состоящего из монокристаллов нанометровых размеров (т.е. нанокристаллического алмаза) [9]. Это связано с тем, что для повышения чувствительности фотодетектора желательно снижение сопротивления алмаза протеканию электрического тока, т.е. уменьшение толщины алмазной пленки.

При CVD-росте монокристаллического алмаза на монокристаллической алмазной подложке тонкую алмазную пленку при оптимальной для фотодетектора вертикальной геометрии протекания тока можно создать лишь путем нанесения на верхнюю поверхность слоя CVDалмаза металлического контакта, стравливания подложки и нанесения на нижнюю поверхность этого слоя другого металлического контакта. Однако необходимое при этом глубокое травление алмаза связано со значительными технологическими трудностями. Поэтому представляет интерес другая технология изготовления тонких алмазных пленок, заключающаяся в CVD-росте поликристаллического алмаза непосредственно на нижнем металлическом контакте, на поверхности которого размещаются „затравочные“ монокристаллы алмаза нанометровых размеров [9], и последующем нанесении на поверхность полученной таким образом нанокристаллической алмазной пленки верхнего металлического контакта. Толщина такой нанокристаллической пленки определяется временем CVD-процесса и поэтому может быть легко сделана достаточно малой. 


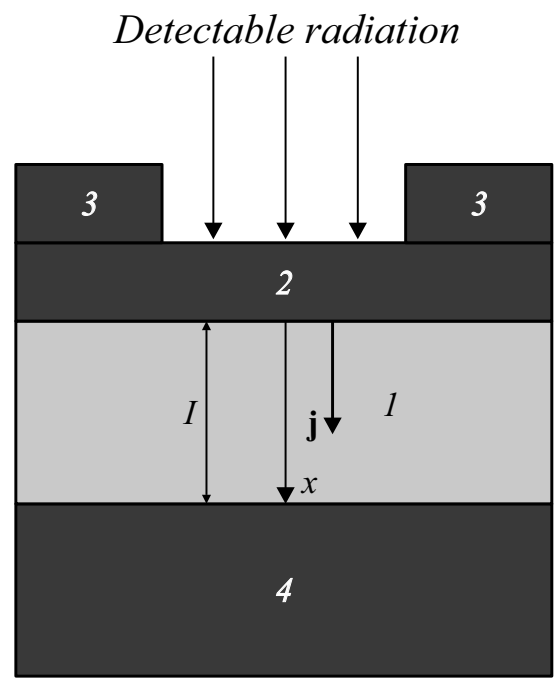

Рис. 1. Схема фотодетектора: 1 - нанокристаллическая алмазная пленка, 2 и 3 - тонкий слой металла и контакты к нему (вместе образующие верхний фоточувствительный контакт), $4-$ нижний толстый металлический контакт, $j-$ полная плотность электрического тока. Также показана используемая система координат.

В связи с этим актуальной является задача расчета вольт-амперной характеристики фотодетектора вертикальной геометрии протекания тока на нанокристаллической алмазной пленке, чему и посвящена настоящая работа.

\section{1. Метод расчета вольт-амперной характеристики}

При расчете вольт-амперной характеристики фотодетектора вертикальной геометрии протекания тока на нанокристаллической алмазной пленке (рис. 1) толщина слоя нанокристаллического алмаза $l$ считается малой по сравнению с его латеральными размерами, так что все величины зависят лишь от координаты $x$ вдоль оси, направленной поперек этого слоя от верхнего фоточувствительного контакта к нижнему, и задача является одномерной. Процесс генерации детектируемым излучением дырок в верхнем фоточувствительном металлическом контакте и их диффузии к слою нанокристаллического алмаза здесь обсуждаться не будет (см. его качественное рассмотрение в [8]), а влияние детектируемого излучения будет моделироваться направленной вдоль оси $x$ плотностью тока $j_{\mathrm{ph}}$, являющейся обусловленной этим излучением добавкой к плотности „темнового“ тока, текущего из верхнего металлического контакта в нанокристаллическую алмазную пленку.

В случае, когда характерный масштаб изменения концентрации дырок $p, p /|d p / d x|$ больше их длины свободного пробега (для достаточно малых размеров нанокристаллов и, следовательно, достаточно малой длины свободного пробега дырок, определяемой рассеянием на их границах, это условие выполняется, см. ниже), плотность направленного вдоль оси электрического тока $j$ в нанокристаллической алмазной пленке дается формулой

$$
j=e p \mu E-e D d p / d x .
$$

Здесь $e>0$ - равный элементарному заряд дырки, $\mu$ - подвижность дырок, $E-$ направленное вдоль оси $x$ электрическое поле $(E>0), D-$ коэффициент диффузии дырок. При умеренной концентрации допирующей акцепторной примеси атомов бора, рассматриваемой ниже, дырки являются невырожденными, так что коэффициент диффузии выражается через подвижность, согласно соотношению Эйнштейна $D=\mu k_{\mathrm{B}} T / e[10]$, где $k_{\mathrm{B}}-$ постоянная Больцмана, $T-$ температура.

Производная электрического поля по координате дается уравнение Пуассона

$$
d E / d x=4 \pi e\left(p-N_{a}^{-}\right) / \varepsilon,
$$

где $N_{a}^{-}$- концентрация отрицательно заряженных атомов-акцепторов (полная концентрация которых, равная сумме концентраций отрицательно заряженных и нейтральных атомов-акцепторов, предполагается однородной и равной $\left.N_{a}\right), \varepsilon \approx 5.7-$ статическая диэлектрическая проницаемость алмаза.

Для решения системы уравнений (1) и (2) относительно $p$ и $E$ необходимо знать функцию $N_{a}^{-}(x)$. В алмазе в отличие от обычных полупроводников с малыми (по сравнению с тепловой энергией при комнатной температуре) энергиями связи дырок на акцепторных уровнях эта функция не является константой. Это связано с тем, что в алмазе энергия связи дырки на акцепторном уровне атома бора во много раз превышает тепловую энергию при комнатной температуре. В результате для алмаза система уравнений (1) и (2) становится более сложной, чем для обычных полупроводников с мелкими акцепторными уровнями. Однако $N_{a}^{-}$может быть выражена через $p$ с помощью простой формулы, получаемой следующим образом.

Число происходящих в единице объема в единицу времени актов рекомбинации дырок с отрицательно заряженными атомами акцепторной примеси пропорциональна произведению их концентраций $p N_{a}^{-}$. В рассматриваемой стационарной ситуации оно должно равняться аналогичному числу актов отдачи нейтральными атомами-акцепторами дырок в валентную зону, которое пропорционально концентрации таких атомов $N_{a}-N_{a}^{-}$. Таким образом, $p N_{a}^{-}=\beta\left(N_{a}-N_{a}^{-}\right)$. Имеющий размерность концентрации коэффициент пропорциональности $\beta$ легко найти, если написать условие равенства этих двух чисел в состоянии термодинамического равновесия (т.е. при $j, j_{\mathrm{ph}}=0$, когда можно ввести однородный в пространстве химический потенциал дырок), в котором концентрации дырок и отрицательно заряженных и нейтральных атомов-акцепторов есть $p_{0}, N_{a 0}^{-}$и $N_{a}-N_{a 0}^{-}$соответственно. Таким образом, он равняется 
$p_{0} N_{a 0}^{-} /\left(N_{a}-N_{a 0}^{-}\right)$. Важно отметить, что для рассматриваемого случая невырожденных дырок, как легко убедиться с помощью стандартных формул для концентрации дырок и отрицательно заряженных акцепторных атомов, приведенных, например, в [10], этот коэффициент не зависит от разности энергии края валентной зоны и химического потенциала дырок. Поэтому он одинаков для всех точек области пространственного заряда в нанокристаллической алмазной пленке и равен

$$
\beta=\frac{1}{\sqrt{2}}\left(\frac{k_{\mathrm{B}} T}{\pi \eta^{2}}\right)^{3 / 2} \frac{m_{d s}^{3 / 2} \exp \left[-E_{a} /\left(k_{\mathrm{B}} T\right)\right]}{4+2 \exp \left[-E_{s o} /\left(k_{\mathrm{B}} T\right)\right]} .
$$

Здесь $\eta-$ постоянная Планка,

$$
m_{d s}=\left\{m_{h h}^{3 / 2}+m_{l h}^{3 / 2}+\exp \left[-E_{s o} /\left(k_{\mathrm{B}} T\right)\right] m_{s o}^{3 / 2}\right\}^{2 / 3}
$$

- эффективная масса дырок, используемая для вычисления плотности их состояний в валентной зоне, $m_{h h, l h, s o}-$ массы тяжелых, легких и спин-отщепленных дырок, равные соответственно $0.588,0.303$ и 0.394 массам свободного электрона $[11], E_{a}-$ энергия связи дырки на акцепторных уровнях атомов бора, зависящая от их концентрации, согласно эмпирической формуле $E_{a}=E_{a 0}-a N_{a}^{1 / 3}$, где $E_{a 0} \approx 0.37 \mathrm{eV}$, $a \approx 10^{-7} \mathrm{eV} / \mathrm{cm}$ [12], $E_{\text {so }} \approx 6 \mathrm{meV}$ - энергия спинорбитального расщепления в алмазе [11], выражение в знаменателе третьего множителя в (3) учитывает вырожденность состояний в подзонах тяжелых, легких и спин-отщепленных дырок валентной зоны по спину и, следовательно, вырожденность формируемых этими подзонами акцепторных уровней. В результате концентрация отрицательно заряженных акцепторных атомов выразится через концентрацию дырок с помощью простой формулы $N_{a}^{-}=N_{a} /(1+p / \beta)$.

Выражая из (2) $p$ через $d E / d x$ и поставляя в (1), можно получить дифференциальное уравнение второго порядка относительно $E$. Однако в него войдут сложные комбинации корней из алгебраических функций от $d E / d x$, вычисление которых ведет к значительным ошибкам. По этой и, возможно, другим причинам процедура численного решения этого уравнения оказывается неустойчивой. Поэтому целесообразно в качестве неизвестной функции выбрать $p$. Для получения уравнения для нее следует продифференцировать (1) по $x$ (с учетом того, что при рассматриваемой ниже достаточно малой концентрации акцепторов $\mu$ определяется рассеянием на границах нанокристаллов алмаза, образующих макроскопически-однородную пленку, и потому не зависит от $x$ ) и подставить в получающееся таким образом соотношение $E$ и $d E / d x$ в виде функций $p$, найденных из (1) и (2) соответственно. Это дает

$$
\begin{aligned}
\frac{d^{2} p}{d x^{2}}= & \frac{e}{k_{\mathrm{B}} T}\left[\frac{d p}{d x} \frac{j+k_{\mathrm{B}} T \mu d p / d x}{e \mu p}\right. \\
& \left.+\frac{4 \pi e p}{\varepsilon}\left(p-\frac{N_{a}}{1+p / \beta}\right)\right] .
\end{aligned}
$$

Граничные условия для (4), $p(0)$ и $p(l)$, находятся из соотношений $j=e p_{0}(0) v_{T} / 4-e p(0) v_{T} / 4+j_{\mathrm{ph}}$ и $j=e p(l) v_{T} / 4-e p_{0}(l) v_{T} / 4$, которые выражают равенство плотностей дырочных токов на границах нанокристаллической алмазной пленки заданному значению $j$. Здесь $p_{0}(0)$ и $p_{0}(l)$ - равновесные (т.е. отвечающие $\left.j, j_{\mathrm{ph}}=0\right)$ концентрации дырок на границах пленки, $e p_{0}(0) v_{T} / 4+j_{\mathrm{ph}}$ и $-e p_{0}(l) v_{T} / 4$ моделируют плотности дырочных токов, текущих соответственно из верхнего и нижнего металлических контактов в пленку [13], - ер $(0) v_{T} / 4$ и ер $(l) v_{T} / 4$ - плотности дырочных токов, текущих из пленки в соответственно верхний и нижний металлические контакты [13], $v_{T}=\sqrt{8 k_{\mathrm{B}} T /\left(\pi m_{v}\right)}-$ средняя тепловая скорость дырок,

$$
\begin{aligned}
m_{v}= & \left\{m_{h h}^{3 / 2}+m_{l h}^{3 / 2}+\exp \left[-E_{s o} /\left(k_{\mathrm{B}} T\right)\right] m_{s o}^{3 / 2}\right\}^{2} / \\
& \left\{m_{h h}+m_{l h}+\exp \left[-E_{s o} /\left(k_{\mathrm{B}} T\right)\right] m_{s o}\right\}^{2}
\end{aligned}
$$

- их эффективная масса. При этом считается, что плотность дырочного тока, текущего из верхнего металлического контакта в нанокристаллическую алмазную пленку, есть сумма ее равновесного значения $е p_{0}(0) v_{T} / 4$ (которое, очевидно, равно взятой с обратным знаком плотности равновесного тока, текущего из пленки в верхний металлический контакт) плюс указанная выше добавка $j_{\mathrm{ph}}$, связанная с генерацией неравновесным детектируемым излучением дырок в верхнем металлическом контакте, а плотность дырочного тока, текущего из нижнего металлического контакта в пленку, есть ее равновесное значение $-e p_{0}(l) v_{T} / 4$ (которое, очевидно, равно взятой с обратным знаком плотности равновесного тока, текущего из пленки в нижний металлический контакт). Равновесные концентрации $p_{0}(0)$ и $p_{0}(l)$ будут считаться одинаковыми (вследствие идентичности химических составов левого и правого металлических контактов) и равными $p_{0}(+\infty) \exp \left[-E_{b} /\left(k_{\mathrm{B}} T\right)\right]$, где $p_{0}(+\infty)$ - концентрация дырок в алмазе за пределами примыкающей к границе с металлом области пространственного заряда (равная вследствие условия зарядовой нейтральности соответствующей концентрации отрицательно заряженных акцепторов $\left.N_{a 0}^{-}(+\infty)\right), E_{b}$ - высота потенциального барьера для дырок на границе алмаза с металлом.

Поскольку сама величина $x$ в (4) не входит, для его решения удобно рассматривать производную $d p / d x$ как функцию $p$. Тогда вторая производная $d^{2} p / d x^{2}$ в левой части (4) примет вид $\frac{d p}{d x} \frac{d}{d p} \frac{d p}{d x}$ и (4) станет дифференциальным уравнением первого порядка относительно $d p / d x$ как функции $p$.

Для численного решения преобразованного таким образом (4) нужно отдельно рассмотреть случаи достаточно малых по сравнению с $j_{\text {ph }}$ (численный критерий малости для конкретных параметров нанокристаллической алмазной пленки и металлических контактов будет

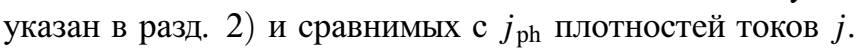

В первом случае начальное условие в задаче Коши нужно ставить в некоторой точке $\bar{p}(\bar{p}>p(0), p(l))$, 
в которой $d p / d x=0$ и координата $\bar{x}$ которой лежит внутри нанокристаллической алмазной пленки, т. е. $0<\bar{x}<l$. Сама величина $\bar{p}$ находится из очевидного равенства

$$
\int_{p(0)}^{\bar{p}} d p /(d p / d x)+\int_{\bar{p}}^{p(l)} d p /(d p / d x)=l,
$$

в котором $d p / d x>0$ при $p(0)<p<\bar{p}$ и $d p / d x<0$ при $\bar{p}>p>p(l)$. Далее от неизвестной функции $d p / d x$ удобно перейти к неизвестной функции $(d p / d x)^{2}$ (для того чтобы избежать бесконечно большого значения $\frac{d}{d p} \frac{d p}{d x}$ в точке $\left.\bar{p}\right)$, так что левая часть преобразованного указанным выше образом уравнения (4) примет вид

$$
\frac{1}{2} \frac{d}{d p}\left(\frac{d p}{d x}\right)^{2}
$$

и решать его отдельно на интервалах $p(0)<p<\bar{p}$ и $\bar{p}>p>p(l)$. При этом, согласно сказанному выше, в левой части (4) следует положить $d p / d x=\sqrt{(d p / d x)^{2}}$ при $\quad p(0)<p<\bar{p} \quad$ и $\quad d p / d x=-\sqrt{(d p / d x)^{2}} \quad$ при $\bar{p}>p>p(l)$. Для определения же разности потенциалов на фотодетекторе

$$
\begin{aligned}
\phi(l)-\phi(0)= & -\int_{0}^{l} E d x=-\int_{p(0)}^{\bar{p}} E d p /(d p / d x) \\
& -\int_{\bar{p}}^{p(l)} E d p /(d p / d x)
\end{aligned}
$$

нужно выразить $E$ через $p$ и $d p / d x$ из (1) и использовать найденную из (4) зависимость $d p / d x$ от $p$.

Во втором случае точки $\bar{p}$ не существует, и производная $d p / d x>0$ во всем интервале $p(0)<p<p(l)$ изменения концентрации дырок в нанокристаллической алмазной пленке. Тогда начальное условие задачи Коши нужно ставить на какой-либо границе этого интервала, а его значение находить из равенства

$$
\int_{p(0)}^{p(l)} d p /(d p / d x)=l .
$$

При этом разность потенциалов на фотодетекторе определится по формуле

$$
\phi(l)-\phi(0)=-\int_{0}^{l} E d x=-\int_{p(0)}^{p(l)} E d p /(d p / d x) .
$$

\section{2. Результаты и их обсуждение}

Пусть толщина слоя нанокристаллической алмазной пленки $l=100 \mathrm{~nm}$, а характерный размер об-

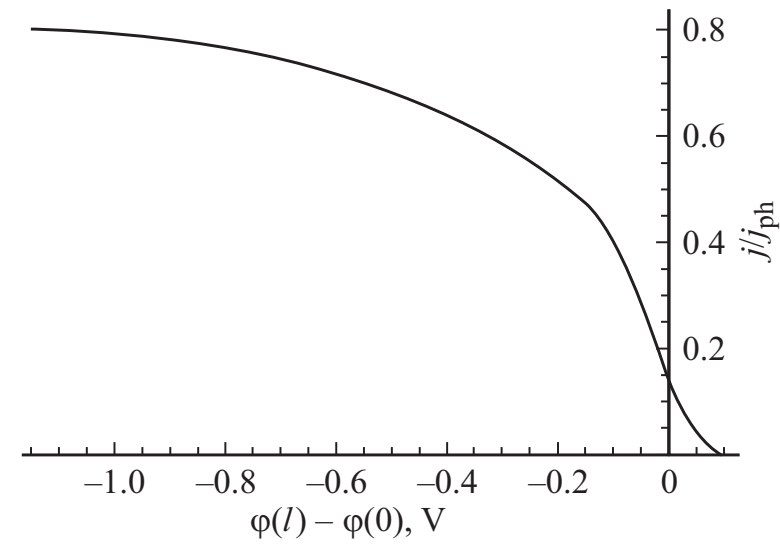

Рис. 2. Вольт-амперная характеристика фотодетектора на нанокристаллической алмазной пленке.

разующих ее нанокристаллов $l_{n c}=5 \mathrm{~nm}$. Концентрация акцепторных атомов бора в этой пленке принимается однородной и составляет $10^{16} \mathrm{~cm}^{-3}$. Температура образца равна комнатной $(T=300 \mathrm{~K})$. Тогда $p_{0}(+\infty)=N_{a 0}^{-}(+\infty) \approx 2.25 \cdot 10^{14} \mathrm{~cm}^{-3}$. При этом, как показывают расчеты, за пределами примыкающей к границе с металлом области пространственного заряда разность энергии края валентной зоны и химического потенциала более чем в 10 раз превышает тепловую энергию $k_{\mathrm{B}} T$ (а при приближении к этой границе эта разность становится еще больше), так что дырки действительно являются невырожденными. Высота потенциального барьера для дырок на границе нанокристаллической алмазной пленки с металлом (зависящая от технологического процесса формирования этой границы) считается равной $0.5 \mathrm{eV}$. Тогда $p_{0}(0)=p_{0}(l) \approx 9 \cdot 10^{5} \mathrm{~cm}^{-3}$. Для $j_{\mathrm{ph}}$ принимается характерное для фотодетекторов на алмазе значение $20 \mu \mathrm{A} / \mathrm{cm}^{2}$ при потоке мощности излучения в видимом диапазоне длин волн порядка $10 \mathrm{~W} / \mathrm{cm}^{2}$ [8]. Подвижность дырок $\mu$ вычисляется по длине их свободного пробега (при рассматриваемой малой концентрации акцепторов, определяемой рассеянием на границах нанокристаллов алмаза, и потому по порядку величины, равной $l_{n c}$ ) по формуле $\mu=e l_{n c} /\left(m_{v} v_{T}\right) \approx 120 \mathrm{~cm}^{2} /(\mathrm{V} \cdot \mathrm{s})$.

При данных параметрах преобразованное указанным выше образом уравнение (4) решалось явным методом Рунге-Кутта. Проведенные расчеты показали, что отношение длины свободного пробега дырок $l_{n c}$ к характерному масштабу изменения их концентрации $p /|d p / d x|$ меньше единицы при всех рассмотренных ниже значениях $j$, что является обоснованием применимости (1) для описания протекания электрического тока через нанокристаллический алмазный фотодетектор.

На рис. 2 приведена вольт-амперная характеристика рассматриваемого фотодетектора на нанокристаллической алмазной пленке. Из него видно, что при $\varphi(l)-$ $-\varphi(0) \approx 0.1 \mathrm{~V}$ (напряжение ,холостого хода“) $j=0$, при $\varphi(l)-\varphi(0)=0 \mathrm{~V}$ имеем $j \approx 0.14 j_{\mathrm{ph}}=2.8 \mu \mathrm{A} / \mathrm{cm}^{2}$ 


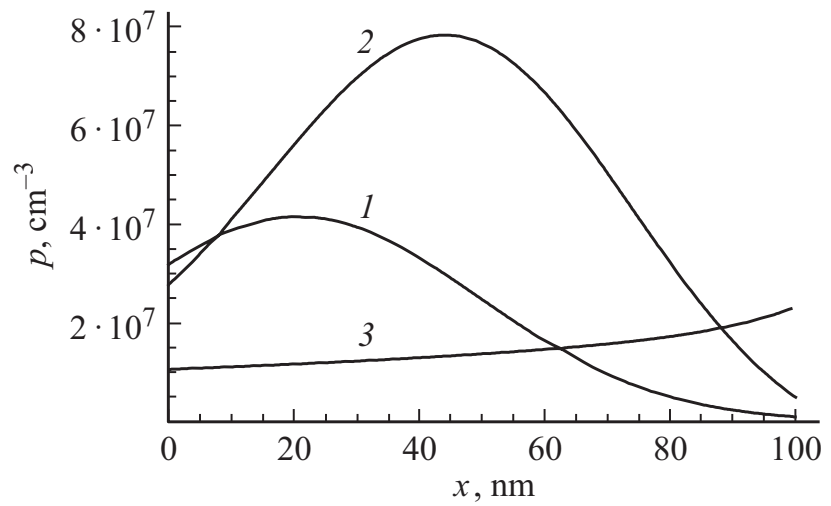

Рис. 3. Концентрация дырок $p$ как функция $x$ при $j=0$ (1), $0.14 j_{\mathrm{ph}}(2)$ и $0.7 j_{\mathrm{ph}}(3)$.

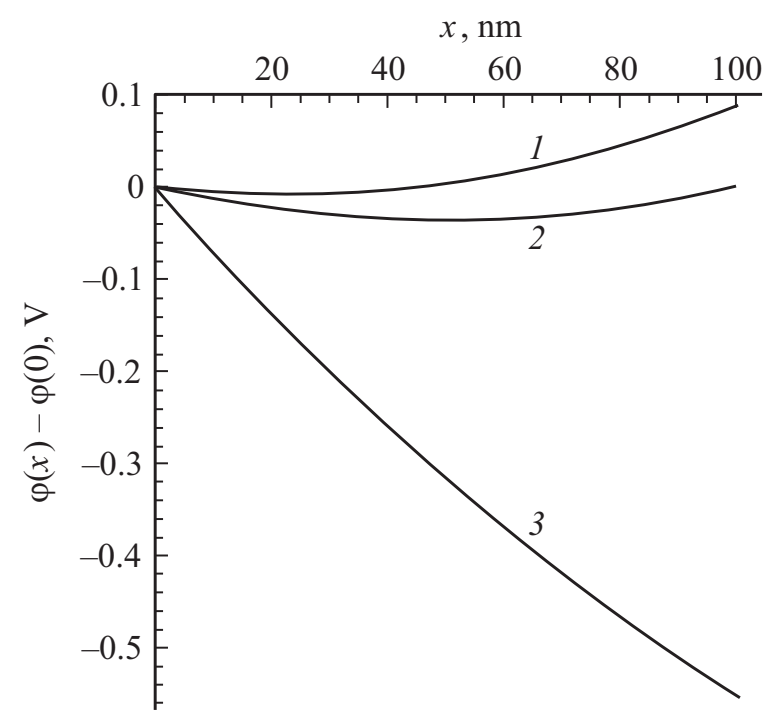

Рис. 4. Разность электростатических потенциалов внутри нанокристаллической алмазной пленки и на ее верхней границе, $\varphi(x)-\varphi(0)$, как функция $x$ при $j=0(1), 0.14 j_{\mathrm{ph}}(2)$ и $0.7 j_{\mathrm{ph}}(3)$.

(плотность тока „короткого замыкания“), точка $\bar{p}$ исчезает при $j \geq 0.7 j_{\mathrm{ph}}$, и при $\varphi(l)-\varphi(0) \rightarrow-\infty$ величина $j \rightarrow j_{\mathrm{ph}}+e p_{0}(0) v_{T} / 4 \approx 1.03 j_{\mathrm{ph}}$ (плотность тока насыщения, получающаяся из граничного условия на верхнем контакте при $p(0)=0)$.

На рис. 3 приведена концентрация дырок в нанокристаллической алмазной пленке в зависимости от координаты $x$ при различных значениях $j$. Из него видно, что при $j=0$ точка $\bar{x} \approx 21 \mathrm{~nm}$, т.е. смещена к верхнему контакту относительно середины нанокристаллической алмазной пленки, а $\bar{p} \approx 4.25 \cdot 10^{7} \mathrm{~cm}^{-3}$, при $j \approx 0.14 j_{\text {рh }}$ имеем $\bar{x} \approx 44.2 \mathrm{~nm} \mathrm{и} \bar{p} \approx 7.83 \cdot 10^{7} \mathrm{~cm}^{-3}$, т. е. $\bar{x}$ удаляется от верхнего контакта, а $\bar{p}$ увеличивается по сравнению со своими значениями при $j=0$, а при $j \approx 0.7 j_{\mathrm{ph}}$ точка $\bar{p}$ отсутствует и зависимость $p(x)$ монотонна.
Электростатический потенциал в нанокристаллической алмазной пленке (относительно его значения при $x=0)$ при различных значениях $j$ показан на рис. 4 . Из него видно, что с ростом плотности тока через фотодетектор точка минимума электростатического потенциала смещается по направлению к нижнему контакту; при достаточно большом $j$ (примерно при $j=0.5 j_{\mathrm{ph}}$ ) она пропадает и потенциал становится монотонно спадающим.

\section{Заключение}

Таким образом, разработан и применен для конкретного примера способ расчета вольт-амперной характеристики и других рабочих параметров детектора видимого и ближнего инфракрасного излучения на поликристаллической алмазной пленке, состоящей из монокристаллов нанометровых размеров. Полученные результаты могут быть использованы при конструировании таких фотодетекторов и оптимизации их характеристик.

Работа выполнена при финансовой поддержке РФФИ (грант 16-02-00450-a).

\section{Список литературы}

[1] Collins A.T. CVD Diamond for Electronic Devices and Sensors / Ed. by R.S. Sussmann. Chichester: Wiley, 2009. P. $165-184$.

[2] Гуляев Ю.В., Чучева Г.В., Набиев А.Э., Алтухов А.А., Попов А.В., Фещенко В.С., Шепелев В.А. // Радиотехника и электроника. 2016. Т. 61. № 4. С. 395398. DOI: 10.7868/S0033849416040069 [Gulyaev Y.V., Chucheva G.V., Nabiev A.E., Altukhov A.A., Popov A.V., Feshchenko V.S., Shepelev V.A. // J. Commun. Techn. Electron. 2016. Vol. 61. N 4. P. 449-452. https://doi.org/10.1134/S1064226916040069]

[3] Ильичев Э.А., Кулешов А.Е., Набиев Р.М., Петрухин Г.Н., Рычков Г.С., Теверовская Е.Г. // Письма в ЖТФ. 2017. Т. 43. Вып. 7. С. 48-55. DOI: 10.21883/PJTF.2017.07.44468.16596 [Ilichev E.A., Kuleshov A.E., Nabiev R.M., Petrukhin G.N., Rychkov G.S., Teverovskaya E.G. // Tech. Phys. Lett. 2017. Vol. 43. P. 345-347. https://doi.org/10.1134/S1063785017040095]

[4] Bruzzi M., Hartjes F., Lagomarsino S., Menichelli D., Mersi S., Miglio S., Scaringella M., Sciortino S. // Phys. Stat. Sol. A. 2003. Vol. 199. N 1. P. 138-144. DOI: https://doi.org/10.1002/pssa.200303816

[5] Majdi S., Kolahdouz M., Moeen M., Kovi K.K., Balmer R.S., Radamson H.H., Isberg J. // Appl. Phys. Lett. 2014. Vol. 105. P. 163510 (1-4). DOI: 10.1063/1.4899278

[6] 3и С. Физика полупроводниковых приборов. М.: Мир, 1984. T. 2. C. 357. [Sze S.M. Physics of Semiconductor Devices. NY:: Wiley, 1981. P. 13.]

[7] Butler J.E., Geis M.W., Krohn K.E., Lawless Jr.J., Deneault S., Lyszczarz T.M., Flechtner D., Wright R. // Semicond. Sci. Technol. 2003. Vol. 18. S67-S71. DOI: https://doi.org/10.1088/0268-1242/18/3/309 
[8] Кукушкин В.А., Радищев Д.Б., Лобаев М.А., Богданов С.А., Здоровейщев А.В., Чунин И.И. // Письма в ЖТФ. 2017. T. 43. Вып. 24. C. 65-71.

DOI: 10.21883/PJTF.2017.24.45343.16860 [Kukushkin V.A., Radischev D.B., Lobaev M.A., Bogdanov S.A., Zdoroveischev A.V., Chunin I.I. // Techn. Phys. Lett. 2017. Vol. 43. N 12. P. $1121-1123$.

DOI: $10.1134 / \mathrm{S} 1063785017120215]$

[9] Williams O.A., Nesládek M., Mareš J.J., Hubík P. Physics and Applications of CVD Diamond / Ed. by S. Koizumi, C.E. Nebel, M. Nesladek. Weinheim: Wiley-VCH Verlag GmbH \& Co.KGaA, 2008. Ch. 2.

[10] Аикрофт Н., Мермин Н. Физика твердого тела. М.: Мир, 1979. Т. 2. Гл. 28, 29. [Ashcroft N.W., Mermin N.D. Solid State Physics. NY:: Holt, Rinehart and Winston, 1976. Ch. 28, 29.]

[11] Pernot J., Volpe P.N., Omnès F., Muret P., Mortet V., Haenen K., Teraji T. // Phys. Rev. B. 2010. Vol. 81. P. 205203 (1-7). DOI: 10.1103/PhysRevB.81.205203

[12] Kukushkin V.A., Snider G., Bogdanov S.A., Chernov V.V. // Phys. Status Solidi RRL. 2014. Vol. 8. N 10. P. 876-879. DOI: $10.1002 /$ pssr.201409302

[13] Ландау Л.Д., Лифииц Е.М. Статистическая физика. Ч. 1. М.: Физматлит, 2001. § 39. [Landau L.D., Lifshitz E.M. Statistical Physics. Part 1. Oxford, UK: Butterworth-Heinemann, 1980. § 39.] 